\title{
A MULTIPLIER RULE FOR A CLASS OF TWO- DIMENSIONAL VARIATIONAL PROBLEMS
}

\author{
BY LAWRENCE M. GRAVES ${ }^{1}$
}

\author{
Communicated by P. R. Halmos, October 3, 1959
}

We consider a calculus of variations problem of minimizing a function $\alpha$ of integrals $J^{i}$ whose integrands $f^{i}$ depend on $x, y$, and functions $w^{j}(x, y), z^{k}(x, y)$, and their first partial derivatives, and possibly also on their mixed second partial derivatives $\partial^{2} w w^{j} / \partial x \partial y, \partial^{2} z^{k} / \partial x \partial y$. The functions $w^{j}$ and $z^{k}$ defining admissible surfaces are restricted to be absolutely continuous in the sense of Vitali, and also in the sense of Tonelli, and moreover to have uniform bounds for the partial derivatives which actually enter. The functions $z^{k}$ are required to take fixed boundary values on the region $G_{2}$ of the $x y$-plane which is the domain of integration. The functions $w^{i}$, on the other hand, are restricted only by assigned initial values on two lines $x=\bar{x}$ and $y=\bar{y}$ which lie in $G_{2}$ or on its boundary. For convenience we may suppose $\bar{x}=\bar{y}=0$. Admissible surfaces are also required to give prescribed values to certain functions $\beta^{m}$ of the integrals $J^{i}$, and to satisfy almost everywhere on $G_{2}$ a set of partial differential equations

$$
g^{p}\left(x, y, w, w_{x}, w_{y}, w_{x y}, z, z_{x}, z_{y}, z_{x y}\right)=0, \quad\left(p=1, \cdots, p_{0}\right) .
$$

The functions $f^{i}$ and $g^{p}$ are supposed to be measurable functions of $(x, y)$, and to have first partial derivatives with respect to their remaining arguments which are uniformly continuous in those arguments and uniformly bounded in a suitable neighborhood of the minimizing surface.

A fundamental assumption for the proofs is that the matrix of partial derivatives of the functions $g^{p}$ with respect to the arguments $w_{x y}^{j}$ has at almost every point $(x, y)$ of $G_{2}$ a minor determinant of order $p_{0}$ whose absolute value is greater than a fixed positive number $\mu$. This assumption excludes the cases treated by Gross [5], Coral [3] and others. However, the multiplier rule below does apply to the minimizing surfaces for the problems considered by Ciliberto [1] and [2], provided the partial derivatives involved are bounded. We also restrict the boundary $G_{2}^{*}$ of $G_{2}$ as follows: the portion of $G_{2}^{*}$ lying interior to the first quadrant is defined by a continuous decreasing function $y=\eta(x)$ on $0<x<a$, and also by a continuous decreasing func-

1 This work was done under Contract No. DA-11-022-ORD-1833 with the Office of Ordnance Research. The proofs of the results reported here are contained in Technical Reports No. 7 and No. 8 issued under this contract. 
tion $x=\xi(y)$ on $0<y<b$. Corresponding restrictions are made for the other quadrants. (These restrictions can be slightly lightened.)

Corresponding to arbitrary multipliers $l^{m},\left(m=0, \cdots, m_{0}\right)$, and $\lambda^{p},\left(p=1, \cdots, p_{0}\right)$, set

$$
F\left(x, y, w, \cdots, z_{x y}, l, \lambda\right)=f^{i} \frac{\partial}{\partial J^{i}}\left(l^{0} \alpha+l^{m} \beta^{m}\right)+\lambda^{p} g^{p},
$$

where the partial derivatives of $\alpha$ and $\beta$ are evaluated for the minimizing values of $J^{i}$. Let the partial derivatives of $F$ with respect to $w^{j}, w_{y}^{j}, w_{x}^{j}, w_{x y}^{j}, z^{k}, z_{y}^{k}, z_{x}^{k}, z_{x y}^{k}$, be denoted respectively by $F_{0 j}, F_{1 j}, F_{2 j}$, $F_{3 j}, F_{4 k}, F_{5 k}, F_{6 k}, F_{7 k}$. Then the multiplier rule may be stated as follows.

Corresponding to each admissible minimizing surface there exist constants $l^{m}$ not all zero and bounded measurable functions $\lambda^{p}(x, y)$ such that along the minimizing surface

$$
\begin{gathered}
F_{0 j}(x, y)+\int_{x}^{\xi(y)} F_{1 j}(s, y) d s+\int_{y}^{\eta(x)} F_{2 j}(x, t) d t \\
+\int_{x}^{\xi(y)} \int_{y}^{\eta(s)} F_{3 j}(s, t) d t d s=0
\end{gathered}
$$

for almost every point $(x, y)$ in the first quadrant, and

$$
\begin{aligned}
F_{4 k}(\bar{x}, \bar{y}) & -F_{4 k}(\bar{x}, y)-F_{4 k}(x, \bar{y})+F_{4 k}(x, y) \\
& -\int_{x}^{\bar{x}}\left[F_{5 k}(s, \bar{y})-F_{5 k}(s, y)\right] d s \\
& -\int_{y}^{\bar{y}}\left[F_{6 k}(\bar{x}, t)-F_{6 k}(x, t)\right] d t \\
& +\int_{x}^{\bar{x}} \int_{y}^{\bar{y}} F_{7 k}(s, t) d t d s=0
\end{aligned}
$$

for almost all intervals $[x, \bar{x}] \times[y, \bar{y}]$ contained in $G_{2}$, with equations corresponding to (1) for the portions of $G_{2}$ in the other quadrants, if any.

When the functions $f$ and $g$ and all the partial derivatives of $f, g$, and the minimizing functions w and $z$ which actually appear are continuous for $(x, y)$ in $G_{2}$, then the multipliers $\lambda^{p}(x, y)$ may be required to be continuous in $G_{2}$, and the equations above will then hold everywhere in $G_{2}$.

The proof given in Technical Report No. 7 is based on methods used by Bliss for one dimensional problems and on the general implicit function theorem of Hildebrandt and Graves. For the special 
case described in the last paragraph, Technical Report No. 8 gives a proof based on the abstract multiplier rule of Goldstine [4] and the Riesz representation theorem for linear functionals.

\section{REFERENCES}

1. Carlo Ciliberto, Su un problema di Mayer per gli integrali doppi, Ricerche Mat. vol. 6 (1957) pp. 205-236.

2. ——, Problemi di Mayer-Lagrange per gli integrali doppi, ibid. vol. 7 (1958) pp. 21-63.

3. Max Coral, The Euler-Lagrange multiplier rule for double integrals, Contributions to the Calculus of Variations, 1931-1932, University of Chicago, 1933.

4. H. H. Goldstine, Conditions for a minimum in abstract space, Illinois J. Math. vol. 2 (1958) pp. 111-123.

5. W. Gross, Das isoperimetrische Problem bei Doppelintegrale, Monatshefte für Mathematik und Physik vol. 27 (1916) pp. 114-120.

University of Chicago 